\title{
Circulation around a thin zonal island
}

\author{
By J. R. WELLS AND K. R. HELFRICH \\ Department of Physical Oceanography, Woods Hole Oceanographic Institution, \\ Woods Hole, MA 02543, USA
}

(Received 15 February 2000 and in revised form 10 September 2000)

Laboratory and numerical experiments are used to study flow of a uniform-density fluid on the $\beta$-plane around a thin zonally elongated island (or ridge segment in the abyss). This orientation is chosen specifically to highlight the roles of the zonal boundary layer dynamics in controlling the circulation around the island. There are examples of deep ocean topography that fall into this category which make the work directly applicable to oceanic flows. Linear theory for the transport around the island and the flow structure is based on a modification of the Island Rule (Pedlosky et al. 1997; Pratt \& Pedlosky 1999). The linear solution gives a north-south symmetric flow around the island with novel features, including stagnation points which divide the zonal boundary layers into eastward and westward flowing zones, and a western boundary layer of vanishing length, and zonal jets. Laboratory experiments agree with the linear theory for small degrees of nonlinearity, as measured by the ratio of the inertial to Munk boundary layer scales. With increasing nonlinearity the northsouth symmetry is broken. The southern stagnation point (for anticyclonic forcing) moves to the eastern tip of the island. The flow rounding the eastern tip from the northern side of the island now separates from the island. Time-dependence emerges and recirculation cells develop on the northern side of the island. Mean transport around the island is relatively unaffected by nonlinearity and given to within $20 \%$ by the modified Island Rule. Numerical solutions of the shallow water equations are in close agreement with the laboratory results. The transition from zonal to meridional island orientation occurs for island inclinations from zonal greater than about $20^{\circ}$.

\section{Introduction}

The presence of large islands (or ridges in the case of abyssal flow) in an oceanic basin introduces several complications into the solution for the flow. Chief among them is the need to determine the streamfunction on the island, or the transport between the island and basin boundary. Godfrey (1989) derived a simple linear formula, known as the Island Rule, to calculate the northward transport east of Australia and thus arrive at an estimate for the Indonesian throughflow. Godfrey's Island Rule assumes a steady linear barotropic flow, a flat bottom and negligible dissipation except within the western boundary layer on the eastern side of the island. This last assumption permitted Godfrey to avoid explicit calculation of the dissipation and to relate the circulation (or streamfunction on the island) to the wind stress distribution to the east of the island. Wajsowicz (1993) sought to account for the complicated geography north of Australia by modifying the Island Rule for the frictional effects that occur when an island is close to a western boundary, for meridional variations in bottom topography and for island chains.

Recently Pedlosky et al. (1997, hereafter referred to as PPSH) took up the problem 
of flow around planetary-scale islands to examine the general validity of the Island Rule beyond the linear regime and, just as importantly, the structure of the flow. They used analytical, numerical and laboratory methods to examine several idealized island configurations including a thin, meridionally elongated island. An interesting consequence of this geometry is the possibility of a closed recirculation cell on the eastern side of the island. Their laboratory and numerical studies confirmed the predictions of linear theory and also showed that with increasing nonlinearity, as measured by the ratio of the inertial to Munk boundary layer width scales, the flow departed significantly from the linear solution. Flow separation and an inertial recirculation cell on the eastern side of the island emerged at moderate nonlinearity. Surprisingly, the linear Island Rule gave robust predictions of the net circulation around the island for even highly nonlinear flows. The issue of multiple thin meridional barriers (a meridional island shadowed by a peninsula, or a second island, to the east) was considered by Helfrich, Pedlosky \& Carter (1999). Again, while the structure of the flow depended upon the degree of nonlinearity, the transport around the island(s) was given by the linear prediction.

PPSH also described situations where the assumptions leading to the Island Rule are violated. One occurs when the zonal extent of the island becomes large enough to make the neglected dissipation in the northern and southern boundary layers comparable to the dissipation in the western boundary layer on the eastern side of the island. They modelled dissipation as either a bottom friction (the Stommel model) or as a lateral friction (the Munk model) and explicitly calculated the dissipation along the zonal boundaries to give a modified Island Rule. A few comparisons with numerical solutions again showed that the modified linear Island Rule gave good estimates of the transport regardless of the nonlinearity of the flow. Pratt \& Pedlosky (1998) generalized the PPSH results to include dissipation on all boundaries of a rectangular island.

In this study we return to the problem of flow around a zonal island to examine the flow structure and transport through laboratory experiments and numerical modelling. The configuration under consideration is a thin zonal island, centrally located and spanning half the diameter of a circular basin. The flow is forced by a spatially uniform wind stress curl. This geometry is chosen specifically to emphasize the diffusive zonal boundary layers on the northern and southern sides of the island and to facilitate exploration of the nonlinear regime with laboratory experiments.

This geometry has direct application in the ocean. Several of the larger islands in the Indonesian Archipelago run nearly east-west. The Hawaiian Island chain is only slightly inclined from the zonal as are the central Aluetian Islands. The Island Rule ideas, described above in terms of wind-driven circulation, are equally applicable to abyssal flow around the topography (e.g. mid-ocean ridge systems, seamount chains and abyssal rises) driven by upwelling. PPSH discussed extension of the Island Rule to stratified flows where the stratification helps to set the ambient potential vorticity distribution. Ridge orientation, which is with respect to isolines of ambient potential vorticity, may be quite different from the geographical orientation. From maps of abyssal potential vorticity (O'Dwyer \& Williams 1997), it is possible to identify deep topographic features that are in this sense zonal. Examples include the Broken Plateau in the Indian Ocean which runs nearly zonally from $85^{\circ} \mathrm{E}$ to $100^{\circ} \mathrm{E}$, the Sala y Gomez Ridge in the South Pacific between $110^{\circ} \mathrm{W}$ to $85^{\circ} \mathrm{W}$ and the SouthWest and SouthEast Indian ridges. Also, the southwest running Walvis Ridge off the coast of Africa parallels the deep potential vorticity contours.

Section 2 contains a discussion of the modified Island Rule and description of the 
linear circulation following PPSH. The laboratory set-up, important parameters and results are in $\S 3$. The laboratory experiments are compared with new solutions of the single-layer shallow-water equations in $\S 4$ along with an analysis of the transition from zonal to meridional orientation. The results are summarized and discussed in $\S 5$.

\section{Theoretical background}

In this section we review the Island Rule and its modification for zonal topography. Details are given in PPSH and Pratt \& Pedlosky (1998). Consider non-divergent barotropic quasi-geostrophic flow in a $\beta$-plane basin which contains an island. The transport between the island and the basin boundary is given by the streamfunction on the island $\psi=\Psi_{I}$, where it is assumed that $\psi=0$ on the basin boundary and the depth $h$ is constant. The circulation around the island is found from integration of the tangential component of the hydrostatic momentum equation around the contour, $C_{I}$, which wraps tightly around the island. This integration gives

$$
\oint_{C_{I}} \boldsymbol{D}(\boldsymbol{u}) \cdot \hat{\boldsymbol{t}} \mathrm{d} s+\oint_{C_{I}} \boldsymbol{T} \cdot \hat{\boldsymbol{t}} \mathrm{d} s=0 .
$$

Here $\boldsymbol{u}(x, y)$ is the horizontal velocity, $\boldsymbol{D}(\boldsymbol{u})$ is the dissipation operator, which may represent linear bottom friction, lateral friction, or both, and $\hat{\boldsymbol{t}}$ is the unit vector tangent to $C_{I}$. The forcing is given by $\boldsymbol{T}$, the wind stress divided by $\rho h$, where $\rho$ is the density. Abyssal flow is forced by the horizontal distribution of vertical pumping (PPSH; Helfrich et al. 1999).

The constraint (1) states that the integrals of the tangential components of forcing and dissipation around the island boundary must balance. This is true even for steady nonlinear flows. In principle, specification of $\boldsymbol{T}$ and $\boldsymbol{D}(\boldsymbol{u})$ is sufficient for determining $\Psi_{I}$. However, this means solving for the boundary layer structure on the island, which in general can be quite difficult. Godfrey (1989) showed that this complication could be overcome with a contour that runs along the western side of the island and continues to the eastern basin boundary along the latitude of the southern end of the island. It then runs up the basin boundary to the latitude of the northern end of the island where it returns to the northwestern corner of the island. This contour avoids the strong dissipation region in the western boundary layer on the eastern side of the island. Dissipation on the western side of the island (an eastern boundary) is insignificant. This leaves only the northern and southern island boundaries. PPSH showed that the relative contribution of these diffusive boundary layers is given by the ratio of the zonal boundary layer thickness scale $\delta_{Z}$ to the meridional extent of the island $y_{n}-y_{s}$,

$$
R_{Z}=\frac{\delta_{Z}}{y_{n}-y_{s}} .
$$

Here $y_{n}$ and $y_{s}$ are the meridional locations of the northern and southern ends of the island, respectively. When dissipation is dominated by lateral friction (i.e. Munk dynamics), PPSH give

where

$$
\delta_{Z}=\left(\delta_{M}^{3} L\right)^{1 / 4},
$$

$$
\delta_{M}=(v / \beta)^{1 / 3}
$$

is the Munk boundary layer width scale, $v$ is the lateral friction coefficient, $\beta$ is the 


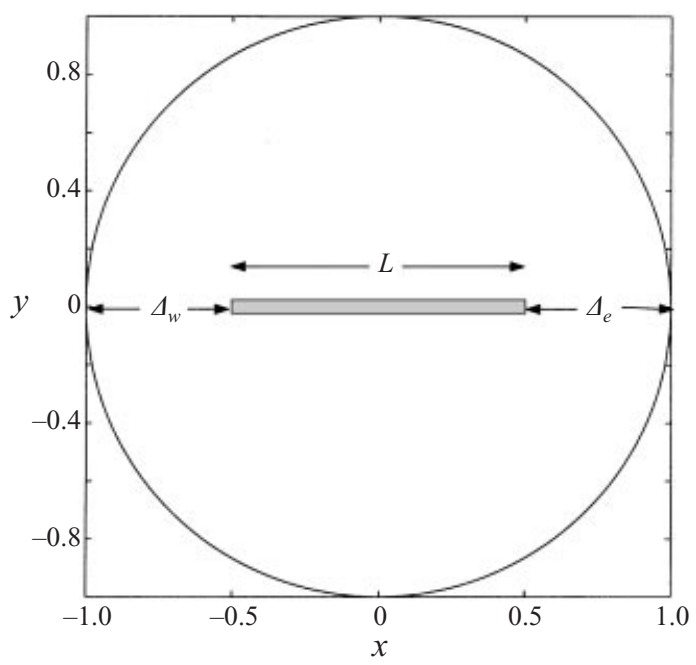

FIGURE 1. Sketch of a thin, zonally elongated island in a circular basin. The island has length $L$ in the zonal, $x$, direction and the gaps between the island and the western and eastern basin boundaries are $\Delta_{w}$ and $\Delta_{e}$, respectively.

meridional gradient of the planetary vorticity, and $L$ is the zonal length of the island. For Stommel dynamics (i.e. bottom friction) PPSH show

$$
\delta_{Z}=\left(\delta_{S} L\right)^{1 / 2},
$$

where the Stommel boundary layer width scale

$$
\delta_{S}=r / \beta
$$

and $r$ is the bottom friction coefficient ( $r^{-1}$ is the Ekman spin-down time).

For $R_{Z} \ll 1$ the zonal boundary layer dissipation is negligible. The assumption of linear flow leads to Godfrey's original Island Rule,

$$
\Psi_{I}=\frac{1}{y_{n}-y_{s}} \int_{y_{s}}^{y_{n}} \psi_{s}\left(x_{i e}(y), y\right) \mathrm{d} y,
$$

where $x_{i e}(y)$ defines the eastern side of the island. The zonal gradient of the Sverdrup streamfunction $\psi_{s}$ is

$$
\frac{\partial \psi_{s}}{\partial x}=\frac{\hat{\boldsymbol{k}} \cdot(\nabla \times \boldsymbol{T})}{\beta}=\frac{W}{\beta} .
$$

The forcing $W$ is defined above and $\hat{\boldsymbol{k}}$ is the vertical unit vector.

For $R_{Z} \geqslant O(1)$ the northern and southern boundary layers cannot be neglected. In the limit of a vanishingly thin, zonal island (figure 1), (1) must be used to find $\Psi_{I}$. For linear flow and dissipation governed by lateral friction PPSH find the modified Island Rule,

$$
\Psi_{I}=\frac{-W}{\beta}\left(\frac{4}{5} L+\Delta_{e}\right)
$$

Here $\Delta_{e}$ is the distance between the eastern tip of the island and the basin boundary. When the dissipation is through Stommel dynamics, the modified Island Rule 


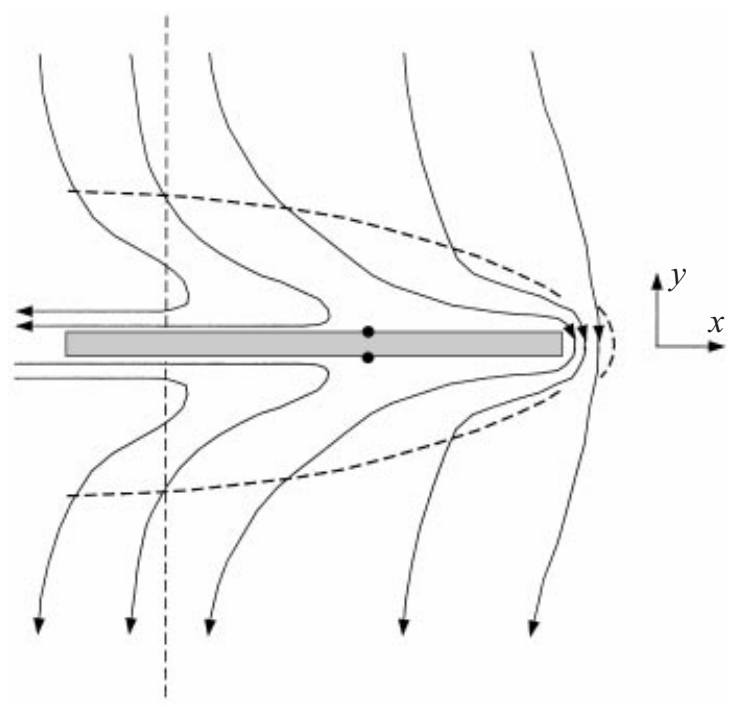

FIGURE 2. Schematic of the linear boundary layer flow around a thin, zonally elongated island of length $L$ (after PPSH). In this case the exterior Sverdrup flow is anticyclonic and approaches the island from the north. The solid circles indicate the predicted stagnation-point locations at $0.6 \mathrm{~L}$ from the western end of the island, and the vertical dashed line at $0.2 L$ indicates the longitude where the net zonal boundary layer transport vanishes. The dashed curves demarcate the Munk boundary layer on the eastern end of the island and the diffusive zonal boundary layers on the northern and southern island boundaries. Positive- $y$ points to the north.

according to PPSH is

$$
\Psi_{I}=\frac{-W}{\beta}\left(\frac{2}{3} L+\Delta_{e}\right)
$$

The quantitative difference between the two is small.

In both cases the island streamfunction $\Psi_{I}$ consists of two parts. One is the Sverdrup transport between the basin boundary and the location of the eastern tip of the island, $\psi_{s}^{I}=-(W / \beta) \Delta_{e}$. Since this is independent of the island, the essential effect of the island on transport is given by the other part, $\Psi_{W}=\Psi_{I}-\psi_{s}^{I}$, the western boundary transport at the eastern tip of the island (less the $O\left(\delta_{M} / \Delta_{e}\right)$ Sverdrup transport within the western boundary layer). For Munk dynamics

$$
\Psi_{W}=\Psi_{I}-\psi_{s}^{I}=-\frac{W}{\beta} \frac{4}{5} L .
$$

Figure 2 illustrates the structure of the flow in the neighbourhood of a thin zonal island within a weak southward Sverdrup flow. The flow is discussed in the context of Munk dynamics, but other than some minor quantitative differences noted below it is the same for Stommel dynamics. The island is bounded by northern and southern diffusive boundary layers which thicken with distance to the west from the eastern tip of the island. At the western end of the island these boundary layers have thickness scaled by $\delta_{Z}=\left(\delta_{M}^{3} L\right)^{1 / 4}$. A western boundary layer of vanishing meridional length and zonal width of $\delta_{M}$ exists at the eastern tip of the island. Some of the fluid encountering the island from the north enters the northern boundary layer to flow east. It then rounds the eastern tip of the island in this western boundary layer conduit (without separating from the island) to enter the southern boundary layer. There it flows west before re-emerging into the southern Sverdrup regime. The rest of the fluid 




FIGURE 3. Streamfunction contours from a numerical model run in the linear regime with $\delta_{I} / \delta_{M}=0.1$. The model is set up to replicate the laboratory experiments. In this plot and subsequent numerical solutions the streamfunction is normalized by the Sverdrup streamfunction at the centre of the basin in the absence of the island. The contour interval is 0.15 . The thick contour has a value equal to the streamfunction on the island. The flow is anticyclonic and positive- $y$ points to the north.

impinging on the northern side of the island flows towards the western end of the island. Once there, it cannot immediately round the island since this is an eastern boundary. It continues west and then slowly turns south and then back east in the westward extension of the diffusive zonal boundary layers and the interior Sverdrup flow. Stagnation points exist on the northern and southern sides of the island. In the Munk model these points are at $\frac{3}{5} L$ from the western tip of the island. However, the transport in the zonal boundary layers is zero at a point $0.2 L$ from the western tip of the island. The flow pattern is symmetric about the $x$-axis and the solution for cyclonic forcing is found by simply inverting the direction of flow in figure 2 .

Figure 3 shows a numerical solution of the single-layer shallow-water equations in a cylindrical $\beta$-plane basin. This anticipates the discussion of the experiments $(\S 3)$ and the numerical model $(\S 4)$, but is introduced here to complete the description of the linear solution and to set the background for the discussion of the experimental results. The figure shows the streamfunction for anticyclonic forcing with a thin island centred in the basin. The length of the island $L$ is equal to the radius of the basin. The strength of the forcing is proportional to the ratio of the inertial to Munk boundary layer scales which is equal to 0.1 (see equations (4), and (16) and (17) below). The flow near the island closely resembles the schematic boundary layer solution sketched in figure 2 . The stagnation points are nearly symmetrically located near the predicted value from the Munk model of $0.6 \mathrm{~L}$ from the western tip. The fluid rounds the eastern tip of the island in a western boundary layer to flow along the southern side of the island. The jagged features near the western basin boundary are due to the rectangular grid discretization and are accentuated by the contouring algorithm.

In PPSH numerical modelling showed that the flow was able to round the eastern tip of a thin island as depicted in figure 3 for very weak forcing. However, for strong forcing the flow became unable to completely round the tip and separated from the island. Further study of this breakdown of the linear theory beyond the 
two numerical examples in PPSH is the focus of the laboratory and numerical work described herein.

\section{Laboratory experiments}

\subsection{Set-up and parameters}

The laboratory experiments are based on the lid-driven, sliced-cylinder analogue of wind-driven circulation on the $\beta$-plane (Pedlosky \& Greenspan 1967; Beardsley 1969). The apparatus used in these experiments is the one used in Helfrich et al. (1999). It consisted of a cylindrical Plexiglas tank of radius $r_{0}=21.4 \mathrm{~cm}$ fitted with a uniformly sloping bottom with slope $s=0.15$. Equivalent north ( $y$ positive) is toward the shallow end of the tank. The mean depth of the fluid $H=20 \mathrm{~cm}$. The island barrier was a vertical $1.2 \mathrm{~cm}$ thick Plexiglas plate centred in the tank and oriented along the $H=20 \mathrm{~cm}$ depth contour. The island spanned the entire depth and had a length $L=21.3 \mathrm{~cm}$. The gaps between the island and the basin's eastern and western boundaries $\Delta_{e}=\Delta_{w}=10.75 \mathrm{~cm}$ (see figure 1 ).

The tank was placed on a turntable which was rotated in a counterclockwise direction (viewed from above) with frequency $\Omega=f_{0} / 2=2 \mathrm{~s}^{-1}$. The flow was forced by a horizontal plate glass lid rotated at frequency $\Delta \Omega$ relative to $\Omega$. The lid was driven by a belt mechanism connected to a stepping motor. The working fluid was either water or a mixture of water and bromothymol blue indicator. Both had kinematic viscosity $v=0.01 \mathrm{~cm}^{2} \mathrm{~s}^{-1}$.

The sloping bottom gives an effective $\beta$-plane, where $f=f_{0}+\beta y$ with

$$
\beta=\frac{2 \Omega s}{H} .
$$

For these experiments $\beta=0.03 \mathrm{~cm}^{-1} \mathrm{~s}^{-1}$. In all runs the lid was driven anticyclonically $(\Delta \Omega<0)$. This results in Ekman pumping at the upper surface, $w_{E}$, given by

$$
w_{E}=\frac{1}{2}(v / \Omega)^{1 / 2}\left(\zeta_{\text {lid }}-\zeta_{\mathrm{g}}\right) .
$$

The vertical vorticity of the lid $\zeta_{\text {lid }}=2 \Delta \Omega$ and $\zeta_{\mathrm{g}}$ is the vertical vorticity of the interior geostrophic flow. The Ekman pumping due to the lid rotation alone,

$$
w_{0}=(v / \Omega)^{1 / 2} \Delta \Omega,
$$

is uniform. When $\Delta \Omega<0$, the downwelling forces an anticyclonic Sverdrup circulation with velocity scale

$$
U=\frac{2 \Omega w_{0}}{\beta H}=\frac{w_{0}}{s},
$$

after using (12).

Viscous dissipation in the laboratory experiments arises either from lateral Laplacian friction with viscosity $v$, or Ekman processes at the top and bottom represented by a drag coefficient $r=2 \Omega E_{v}^{1 / 2}$ in the quasi-geostrophic approximation. The Ekman number $E_{v}=v /\left(\Omega H^{2}\right)$. Lateral friction gives the Munk boundary layer width scale $\delta_{M}$ given in (4). Bottom and lid drag give the Stommel boundary layer width $\delta_{S}$ in (6). For these experiments $\delta_{M}=0.69 \mathrm{~cm}$ and $\delta_{S}=0.48 \mathrm{~cm}$. Both are much smaller than the tank diameter $2 r_{0}$. Since $\delta_{M} / \delta_{S} \sim 1$ the boundary layers are controlled by a combination of lateral and bottom processes. In what follows we will discuss the experimental and numerical results in the context of the Munk model for the Island 
Rule (9). The flow will probably lie between the Stommel and Munk predictions; however, the quantitative differences between the predictions are minor.

For homogeneous, rapidly rotating flow on the $\beta$-plane, nonlinearity is represented by the inertial boundary layer width scale (Pedlosky 1996, § 2.3)

$$
\delta_{I}=(U / \beta)^{1 / 2} .
$$

When viscous processes are controlled by lateral friction the appropriate measure of flow nonlinearity is $\delta_{I} / \delta_{M}$, which can be thought of as a Reynolds number. From (4), (16) and (15)

$$
\frac{\delta_{I}}{\delta_{M}}=\frac{(\Delta \Omega / \Omega)^{1 / 2}}{2^{1 / 6} E_{v}^{1 / 12} s^{2 / 3}} .
$$

For $\delta_{I} / \delta_{M} \geqslant O(1)$ nonlinear effects are expected to dominate.

Other nonlinear influences in the laboratory experiments may arise from the Ekman and Stewartson boundary layers. In the case of the Ekman layers nonlinear influences are negligible provided that the quasi-geostrophic compatibility condition, $E_{v}^{1 / 2} \leqslant O(R o)$, is satisfied (Pedlosky 1987, §4.5). Here $R o$ is the Rossby number. One measure for $R o$ is the imposed lid rotation $|\Delta \Omega / \Omega|$. In the experiments $|\Delta \Omega / \Omega|=0.005-0.1$ and $E_{v}^{1 / 2}=3.54 \times 10^{-3}$. This compatibility condition is easily satisfied. If local flow features develop relative vorticity comparable to $f(R o=O(1))$ nonlinear Ekman pumping effects can become important and a modified Ekman pumping formula may be required (Hart 1995). Similar considerations apply to the sidewall Stewartson layers (Hart 1995). However, both the laboratory and numerical model flows considered herein do not develop $O(1)$ Rossby numbers. The interior flow remains quasi-geostrophic and the nonlinearity of the flow is measured by $\delta_{I} / \delta_{M}$.

The experiments are described by $\delta_{I} / \delta_{M}$ which was varied by changing $\Delta \Omega$. All other parameters were fixed at the values given above. Nominal values $\delta_{I} / \delta_{M}=[0.59$, $0.71,0.81,1.16,1.41,1.63,1.81,2.00,2.16,2.31,2.44,2.56]$ were examined. Runs with $\delta_{I} / \delta_{M}<0.59$ were difficult due to the very weak flows which were easily disturbed by minor thermal gradients in the laboratory, irregularities in the lid flatness or rotation, and irregularities in table rotation.

The experiments were visualized and measured in several ways. Voltage applied to thin stainless steel electrodes in the thymol blue solution produced dye streaks and patches. The flow was also imaged using a laser light sheet to illuminate 150 250 micron pliolite particles suspended in water. In both cases video images were digitized and stored on a PC with sequence capturing software and logged to video tape. For transport measurements an electrode was located at mid-depth spanning the gap east of the island to produce a swath of dye. The differences in area swept by the dye at specified time intervals and the fluid depth were used to determine the transport between the island and the tank boundary and hence $\Psi_{I}$. The images used in this analysis were always prior to development of any fine filaments along the front. Further, time intervals of $4 \mathrm{~s}\left(\delta_{I} / \delta_{M}=2.57\right)$ to $90 \mathrm{~s}\left(\delta_{I} / \delta_{M}=0.59\right)$ were too short for mass diffusion to be significant. Thus the dye front defines a material surface. Combined with the incompressibility and two-dimensionality of the flow, this results in an unambiguous estimate of Eulerian transport between the eastern end of the island and the basin boundary averaged over the time interval of the dye images, despite any unsteadiness of the flow. We estimate experimental errors in measured transport to be $\pm 5 \%$ percent. Three experiments were conducted for most values of $\delta_{I} / \delta_{M}$ and from each run a minimum of three independent estimates of the transport were determined. It is worth noting that other interpretations of 
dye or particle streaks are subject to the usual differences between streaklines and streamlines. For example, locations of stagnation points were determined by placing electrodes adjacent to the island boundaries. From images of streaks, locations of flow convergence and divergence along the island boundary were determined. Only when the local flow was steady were these convergent and divergent points stagnation points.

\subsection{Results}

Flow structure

An overview of the experiments is shown in figure 4 where streak images from runs with $\delta_{I} / \delta_{M}=1.16,1.62,1.98,2.29$ and 2.56 are shown. Note that not all of the tank is visible, though the boundary can be inferred from its location in the bottom left and right corners of each image. At $\delta_{I} / \delta_{M}=1.16$ (figure $4 a$ ) the flow is very slow $\left(U=0.019 \mathrm{~cm} \mathrm{~s}^{-1}\right)$ and the streak image is just at the point of usefulness. Longer exposures were not possible because of particle drift out of the light sheet. However, even at this moderate value of nonlinearity features of the linear theory are clear. The intensified western boundary current on the eastern end of the island is identifiable as is the broader flow to the west of the island. In the immediate vicinity of the island the flow is approximately symmetric in the north-south direction.

As the nonlinearity is increased the symmetry is lost. This is clear for $\delta_{I} / \delta_{M}=1.63$ (figure $4 b$ ). A stationary eddy-like feature is present on the northern boundary. The flow around the eastern end of the island separates to form a jet flowing to the southwest. Further increases in $\delta_{I} / \delta_{M}$ accentuate these features and, while it is not apparent in these images, the flow becomes unsteady for $\delta_{I} / \delta_{M}=1.82$. Unsteadiness first appears as a weak pulsating of the flow limited primarily to the northern half of the basin. For $\delta_{I} / \delta_{M}>2$ the eddy on the northern side of the island appears to propagate to the eastern tip and the formation process repeats. The flow around the western end separates to generate small eddies.

We have not determined if the unsteady flow in the experiments is periodic or irregular, but the numerical runs discussed in the next section show that steady flow gives way to periodic and then to irregular flow with increasing $\delta_{I} / \delta_{M}$. Also note the increasing size of the inertial recirculation cell in the northwest corner of the tank (a source of north-south asymmetry) and the presence of eddies just to the east of this feature. This behaviour agrees with lid-driven sliced-cylinder experiments without barriers (Beardsley 1969; Griffiths \& Kiss 1999) and is due to the inability of the western boundary layer to dissipate sufficient relative vorticity as the fluid flows along the western boundary of the basin (Pedlosky 1996, § 2.9).

Details of the flow for moderate nonlinearity, $\delta_{I} / \delta_{M}=1.16$, are shown in figure 5 . In figure $5(a)$ an electrode north of the island emits seven dye streaks. These streaks show more clearly than figure $4(a)$ the close north-south symmetry and the structure of the zonal boundary layers which broaden with distance from the eastern end of the island. Figure $5(b)$ shows the broad turning flow rounding the western end of the island. The location of the southern stagnation point is near the rightmost extension of the dye. The location of the point where the zonal boundary layer transport vanishes $(0.2 L$ from the western end of the island in the Munk theory) is difficult to determine from the experiments. However, the stagnation points on the northern and southern boundaries are more discernible. For this case the southern stagnation point lies at about $0.77 \mathrm{~L}$ from the western end, slightly farther than the linear theory prediction of $0.6 \mathrm{~L}$. The stagnation point on the northern side of the island is at $0.58 \mathrm{~L}$. The flow through the gap east of the island is shown in figure $5(\mathrm{c})$. The 
(a)

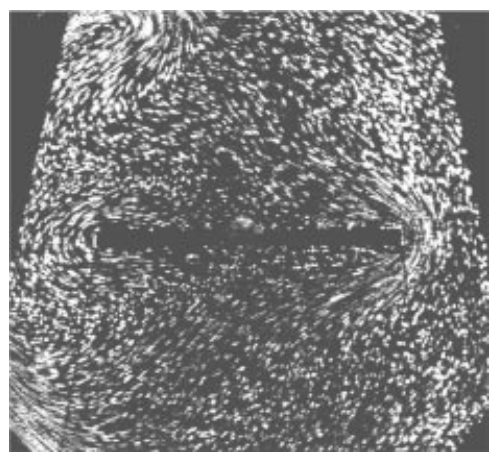

(c)

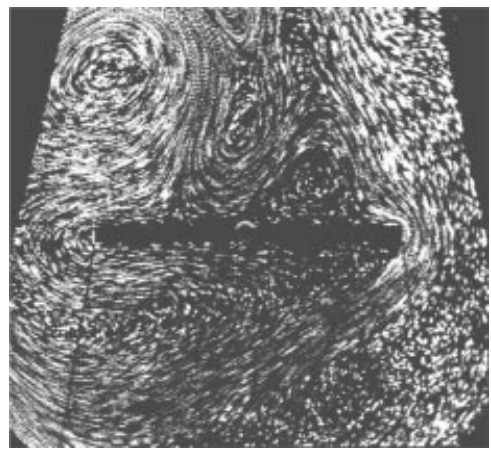

(b)

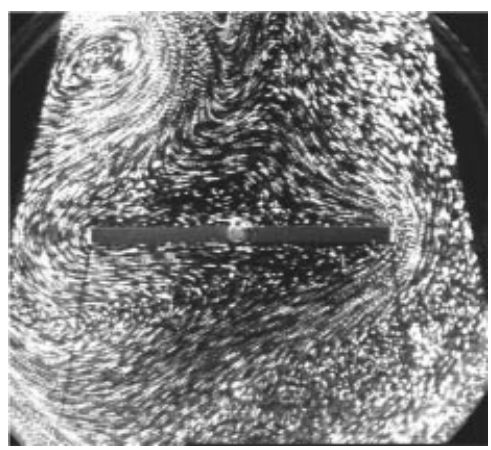

(d)

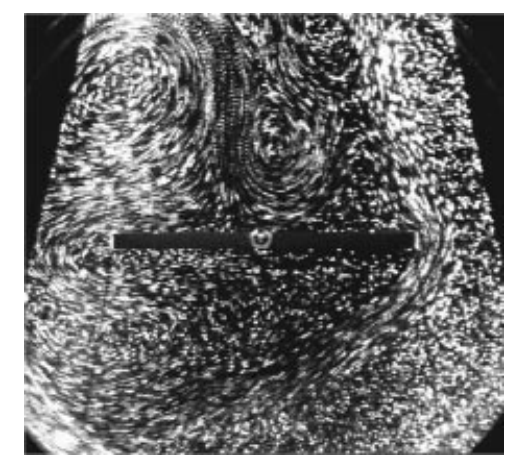

(e)

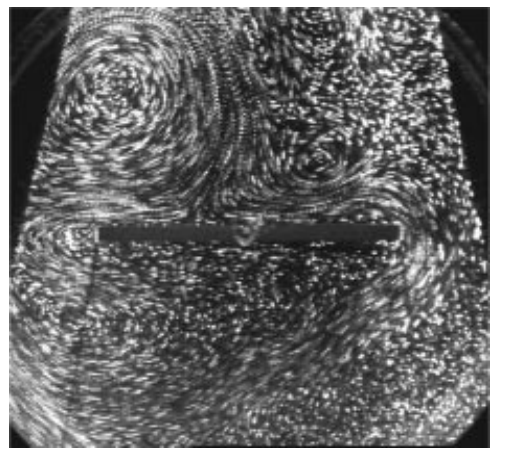

FIGURE 4. Particle streak images for several laboratory experiments. (a) $\delta_{I} / \delta_{M}=1.16,(b) 1.63,(c)$ $1.98,(d) 2.29$ and $(e) 2.57$. The exposure times for these streaks are 10.1, 7.6, 5.1, 3.8 and $3.8 \mathrm{~s}$ for $(a-e)$, respectively. The flow is anticyclonic and the equivalent north is at the top of each image.

intensified western boundary current is clear. The flow appears to separate from the island; however, this is the result of the weak production of dye adjacent to the island, the no-slip boundary condition and the relatively short time of dye emission in figure 5(c). Dye sources along the northern boundary of the island (not shown) clearly show that dye completely rounds the eastern end of the island to flow westward along the southern side. These figures qualitatively validate the linear theory even for this moderately nonlinear case. However, the eastward shift of the southern 



FiguRE 5. Dye streak images for a laboratory experiment with $\delta_{I} / \delta_{M}=1.16$. (a) Flow around the island. $(b)$ Flow through the western gap. (c) Flow through the eastern gap. The equivalent north is at the top of each image. The streak durations are approximately 28 minutes in $(a)$ and 2 minutes in $(b)$ and $(c)$.

stagnation point from the linear prediction of $0.6 \mathrm{~L}$ signals the onset of north-south asymmetry.

Figure 6 shows similar dye swathes in the eastern and western gaps for $\delta_{I} / \delta_{M}=1.82$. The dyed fluid from the western end now extends nearly the complete length of the southern side. At this value of nonlinearity the flow around the eastern end of the island separates. 



FiguRE 6. Dye streak images for a laboratory experiment with $\delta_{I} / \delta_{M}=1.82$. (a) Flow through the western gap. (b) Flow through the eastern gap. The equivalent north is at the top of each image. The streak durations are approximately 1 minute in both frames.

Figure 7 for $\delta_{I} / \delta_{M}=2.57$ shows several new features. First there are disturbances on the intense southwestern jet and these appear in the dye swathes from both the eastern and western gaps (figures $7 a$ and $7 b$ ). Also, just off the western end of the island (figure $7 a$ ), a dark patch of dye is evident as are zonal stripes of dye. These features are due to unsteady eddy production at the western tip and then subsequent straining of the eddy in the southern boundary layer flow. Eddy formation on the northern boundary is shown in figure 7(c). Thymol blue dye streaks from several points just off the northern side of the island have been rolled up into an eddy which has propagated to the the eastern end of the island.

\section{Stagnation-point locations}

The locations of the southern and northern stagnation points were determined from both thymol blue dye and from the particle streak images. The location of the southern stagnation point is easiest to determine with dye since it corresponds to flow convergence, and is shown in figure 8 . For $\delta_{I} / \delta_{M}<1$ the stagnation point is at $0.62 L \pm 0.03 L$ from the western end of the island, which is between the Munk and Stommel model predictions of $0.6 L$ and $0.67 L$, respectively. As $\delta_{I} / \delta_{M}$ is increased, the stagnation point rapidly moves towards the east. When $\delta_{I} / \delta_{M}>1.5$ it is at, or 

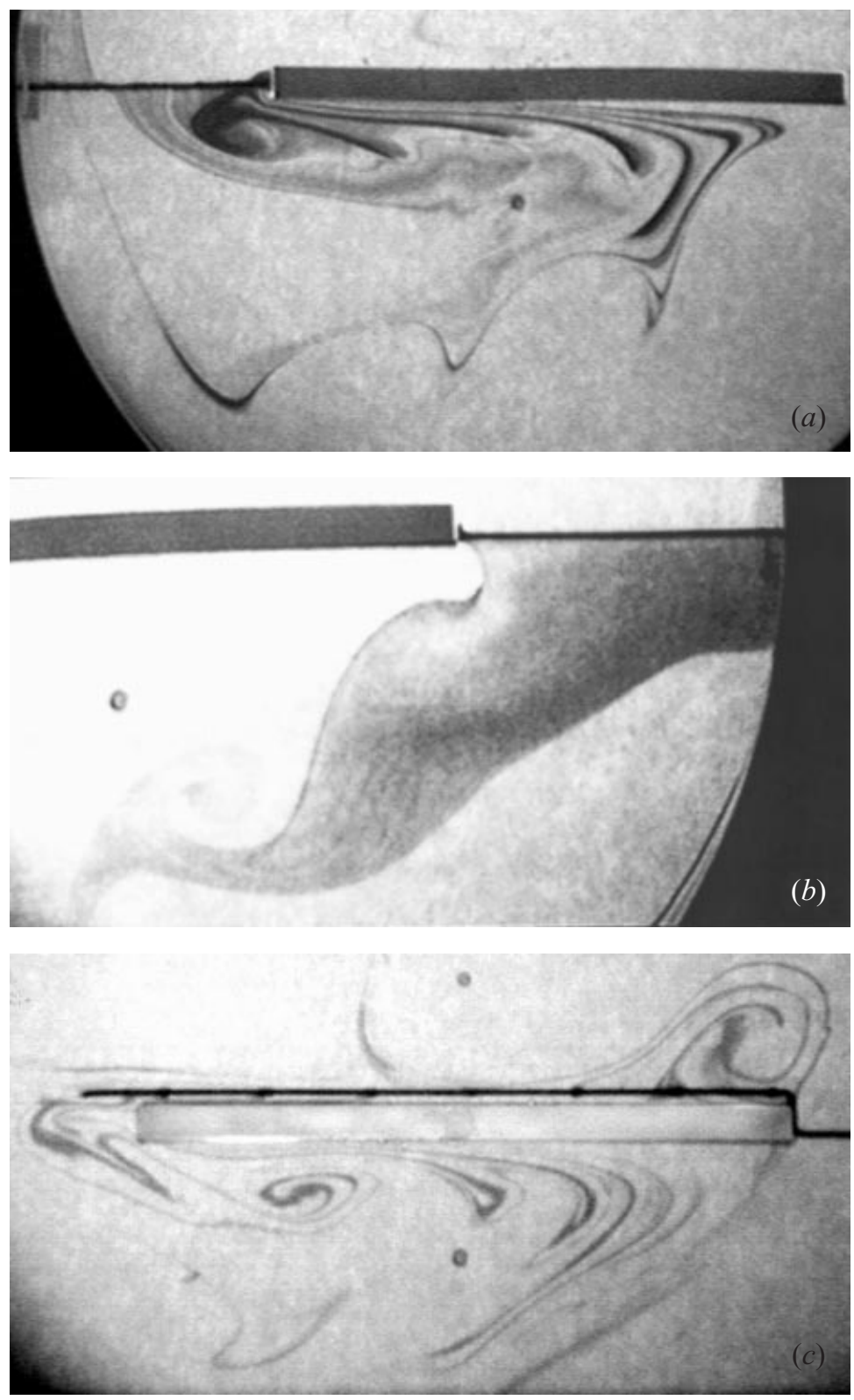

FIGURE 7. Dye streak images for a laboratory experiment with $\delta_{I} / \delta_{M}=2.57$. (a) Flow through the western gap. (b) Flow through the eastern gap. (c) Eddy formation on the northern boundary. The equivalent north is at the top of each image. The streak durations are approximately $40 \mathrm{~s}$ in each frame.

very near, the eastern end indicating separation of the flow rounding the corner. For $\delta_{I} / \delta_{M}>1.63$ the overall flow is unsteady. However, along the southern boundary of the island the flow is essentially steady for all $\delta_{I} / \delta_{M}$. Further, for $\delta_{I} / \delta_{M}>1.63$ the boundary layer flow always separates as it rounds the eastern end of the island. This is denoted in figure 8 by placing the stagnation point at $L=1$, even though formally a stagnation point is not well defined. 


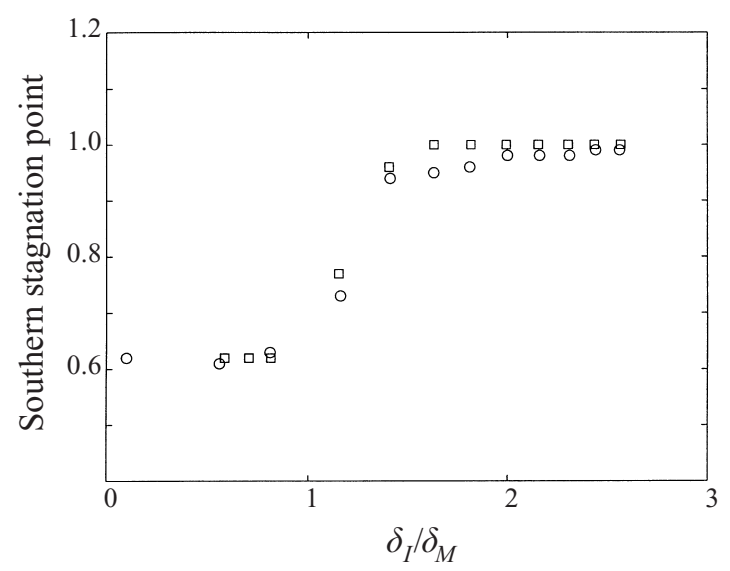

FIGURE 8 . Southern stagnation point location measured from the western end of the island versus $\delta_{I} / \delta_{M}$. The laboratory experiments are indicated by the squares and the circles are from the numerical model runs discussed in $\S 4$.


FIGURE 9. Transport around the island from the laboratory experiments. (a) Transport $T$ normalized by $H \Psi_{I}$ from (9). (b) Transport in the western boundary layer, $T-\psi_{s}^{I}$, normalized by $H \Psi_{W}$ from (11).

The northern stagnation point location is at $0.58 \mathrm{~L} \pm 0.04 \mathrm{~L}$ from the western end of the island for $\delta_{I} / \delta_{M}<1.63$. Once unsteadiness emerges the flow is characterized by eddy formation on the northern boundary and there may be multiple locations of flow convergence or divergence along the boundary (cf. figure $4 c, d$ ).

\section{Transport}

In figure $9(a)$ the measured transport $T$, normalized by the linear prediction $H \Psi_{I}$ from the Munk model (9), is plotted versus $\delta_{I} / \delta_{M}$. In evaluating (9) we use $W=2 \Omega w_{0} / H$ with $w_{0}$ from (14). The plot shows all the individual determinations of the transport. The average transport is $80 \%$ of that from the linear theory with no clear dependence on $\delta_{I} / \delta_{M}$.

A more critical test of the Island Rule is the transport in the western boundary layer, $\Psi_{W}$ from (11), which removes the island-independent Sverdrup contribution. Figure $9(b)$ shows the measured transport $T$ less the Sverdrup transport to the east of the island $H \psi_{s}^{I}=-2 \Omega w_{0} \Delta_{e} / \beta$, normalized by the linear theory $H \Psi_{W}$ from (11). The differences between the theory and experiments increase slightly. The measured boundary layer transport averages about $65-70 \%$ of the predicted value compared to $80 \%$ for the total transport. Again there is little dependence on nonlinearity. 


\section{Numerical modelling}

As a complement to the laboratory experiments we have performed some numerical model calculations for conditions that simulate those in the laboratory. The solutions were carried out with the Miami Isopycnal Coordinate Model (MICOM) described by Bleck et al. (1992). The model solves the shallow-water equations in isopycnal coordinates. MICOM was used in PPSH and as in that study a homogeneous singlelayer version of the model is employed. Further details of the model formulation can be found in PPSH. An advantage of this model is that it has a free surface and thus internally determines the circulation around the island.

Rather than run the model for the precise laboratory parameters (the MICOM model internal set-up made this difficult, e.g. the model is hard-wired for a minimum water depth of $1 \mathrm{~m}$ ) we use a circular $\beta$-plane basin with a radius $r_{0}=1000 \mathrm{~km}$ and mean fluid depth $H=1000 \mathrm{~m}$. The basin contains a centrally located island with $L=r_{0}$. The island thickness was $60 \mathrm{~km}$ (three numerical grid cells) which is just slightly thicker than the laboratory island when scaled by $r_{0}$. The Coriolis parameter $f=f_{0}+\beta y$ with $f_{0}=10^{-4} \mathrm{~s}^{-1}$. The meridional gradient of planetary vorticity $\beta$ was set to $\beta=1.605 \times 10^{-11} \mathrm{~m}^{-1} \mathrm{~s}^{-1}$ to give a variation of $f$ of $0.1605 f_{0}$ when $y=r_{0}$, as in the laboratory experiments. The model lateral friction coefficient (no-slip boundaries) was chosen from (4) so that $\delta_{M}=32 \mathrm{~km}$, which when divided by $r_{0}$ equals the laboratory ratio $\delta_{M} / r_{0}$. The model also has linear bottom drag with drag coefficient $r=\delta_{S} \beta=3.58 \times 10^{-7} \mathrm{~s}^{-1}$, duplicating the laboratory value of $\delta_{S} / \delta_{M}$.

The model is forced by a circular wind stress

$$
\tau=\frac{\tau_{m}}{r_{0}}(\hat{\boldsymbol{y}} y-\hat{\boldsymbol{j}} x),
$$

where $\tau_{m}(>0)$ is the magnitude of the stress and $\hat{\boldsymbol{\imath}}$ and $\hat{\boldsymbol{j}}$ are unit vectors in the $x$-and $y$-directions, respectively. This distribution gives a uniform anticyclonic wind stress curl $\hat{\boldsymbol{k}} \cdot \nabla \times \tau=-2 \tau_{m} / r_{0}$ and a Sverdrup velocity scale $U=2 \tau_{m} / \rho H r_{0} \beta$. The inertial boundary length scale $\delta_{I}$ from (16) is

$$
\delta_{I}=\left(\frac{2 \tau_{m}}{\rho r_{0} H \beta^{2}}\right)^{1 / 2} .
$$

The numerical runs were conducted by varying $\tau_{m}$, with other parameters held fixed, to give $\delta_{I} / \delta_{M}$ corresponding to the laboratory experiments. All the non-dimensional parameters in the governing barotropic vertical vorticity equation, $\delta_{I} / r_{0}, \delta_{M} / r_{0}$ and $\delta_{S} / r_{0}$, are identical in the numerical model and laboratory experiments. The only differences are that the shallow-water model does not resolve the top and bottom Ekman layers and sidewall Stewartson boundary layers present in the experiment. However, as described in $\S 3.1$, nonlinearities associated with the Ekman layers are negligible. Since the flow remains in the quasi-geostrophic regime the mass transport through the geostrophic interior can be consistently ignored. The homogeneous single-layer model captures the essential features of the interior geostrophic flow in the experiments.

\section{Flow structure}

A numerical run for $\delta_{I} / \delta_{M}=0.1$ has already been shown in figure 3. This and all subsequent streamfunction plots are normalized by the magnitude of the Sverdrup streamfunction at the centre of the basin in the absence of topography, $\psi_{s}=2 \tau_{m} / \rho H \beta$. 
(a)



(d)

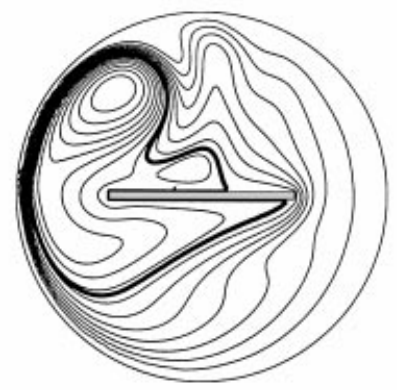

(e) (c)

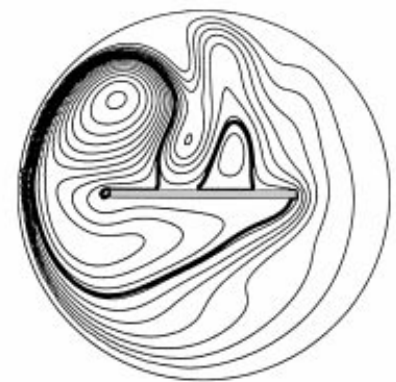



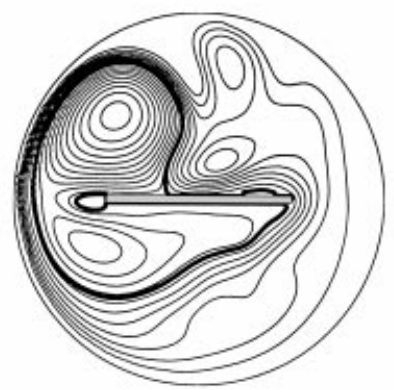

FIGURE 10. Streamfunction contours for numerical experiments with $(a) \delta_{I} / \delta_{M}=1.16,(b) 1.63$, (c) 2.00, $(d) 2.31$ and $(e) 2.56$. The streamfunction is normalized by the Sverdrup streamfunction at the centre of the basin in the absence of the island. The contour interval is 0.15 . The thick contour has a value equal to the streamfunction on the island. North is at the top of each panel.

For those nearly linear conditions the flow around the island is similar to the linear boundary layer prediction.

Figure 10 shows streamfunction plots of the numerical solutions at $\delta_{I} / \delta_{M}=1.16$, $1.63,2.00,2.31$ and 2.56 . These should be compared with the experimental particle streak images at nearly identical values of $\delta_{I} / \delta_{M}$ shown in figure 4 . As in the experiments, increasing $\delta_{I} / \delta_{M}$ results in increasing north-south asymmetry and the emergence of an eddy on the northern boundary of the island. The numerical experiments have steady solutions for $\delta_{I} / \delta_{M} \leqslant 1.63$, periodic solutions for $1.81 \leqslant$ $\delta_{I} / \delta_{M} \leqslant 2.31$ and irregular solutions for $\delta_{I} / \delta_{M} \geqslant 2.44$. The numerical solutions when the flow is unsteady, figures $10(c-e)$, are chosen at a particular phase of the oscillation when they agree best with the corresponding experimental streak images in figure 4 . However, the magnitude of the unsteady effects (discussed more below) is quite small. In general, the qualitative agreement between the experiments in figure 4 and the numerical results in figure 10 is quite good.

The solution at $\delta_{I} / \delta_{M}=2.0$ is illustrated in figures 11 and 12. Figure 11 shows the transport around the island as a function of time scaled by the advective time scale $r_{0} / U$. After some initial transients the transport becomes periodic. Transport fluctuations are only several percent of the mean value. Figure 12 shows the streamfunction at four points during one period of oscillation. The main effect is a pulsing of the strength of the eddy on the north side of the island and a small eddy at the western tip. The northern eddy position is essentially stationary. The flow on the southern side of the island is not greatly affected, and the flow separates at, or very near, the eastern tip. 




FIGURE 11. The transport around the island, normalized by (9), versus time (normalized by $r_{0} / U$ ) for $\delta_{I} / \delta_{M}=2.0$.

(a)

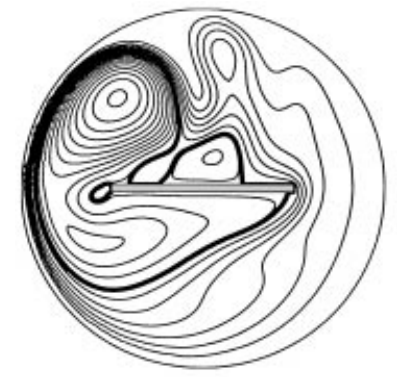

(c)

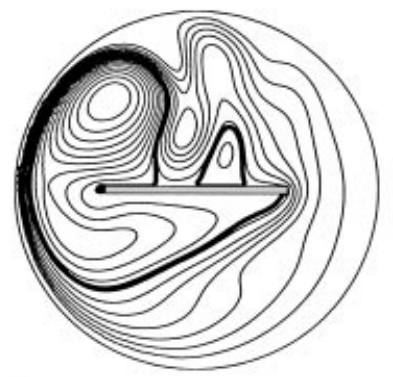

(b)

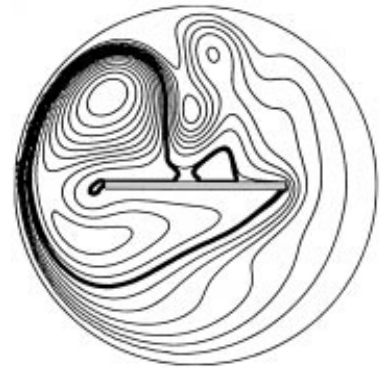

(d)

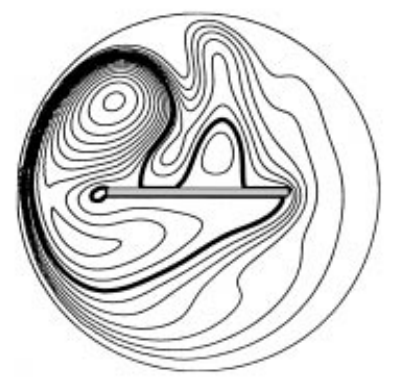

FIGURE 12. The streamfunction for $\delta_{I} / \delta_{M}=2.0$ at four times during an oscillation period of the island transport shown in figure 11: (a) $t=1.835,(b) 1.892,(c) 1.949$ and $(d) 2.007$. The times are normalized by $r_{0} / U$. North is at the top of each panel.

Figures 13 and 14 show similar plots at $\delta_{I} / \delta_{M}=2.56$ when the flow is irregular. Again, the magnitude of the unsteadiness represented by the island transport is relatively small. The streamfunction plots in figure 14 show the propagation of an eddy on the northern boundary of the island towards the eastern tip where it decays. The eddy attached to the western tip of the island is now larger than in figure 12 and may extend along the southern boundary of the island. In the southwestern flowing jet extending from the eastern tip of the island an eddy is evident. These processes continue to repeat with integration time, though with varying period and intensity. These features are similar to the experimental images of dye swaths shown in figure 7. 


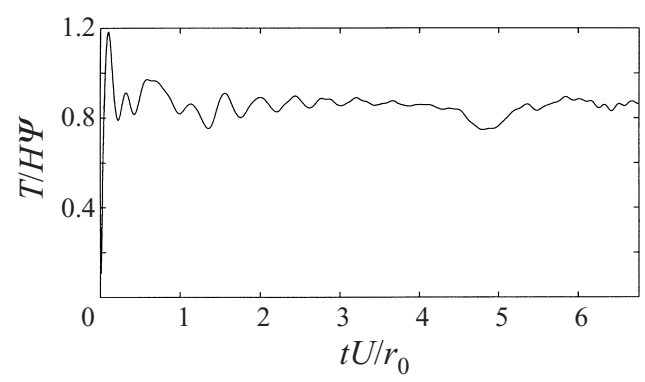

FIGURE 13. The transport around the island, normalized by (9), versus time (normalized by $r_{0} / U$ ) for $\delta_{I} / \delta_{M}=2.56$.

(a)

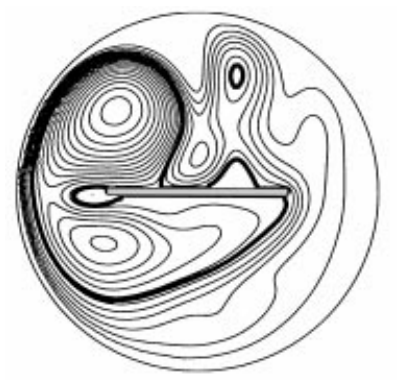

(c)

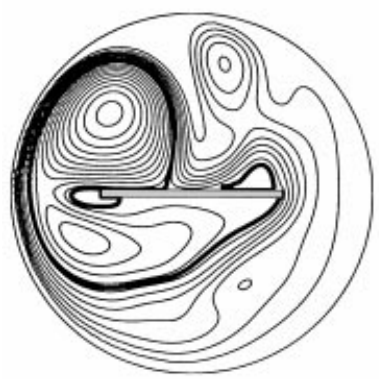

(b)

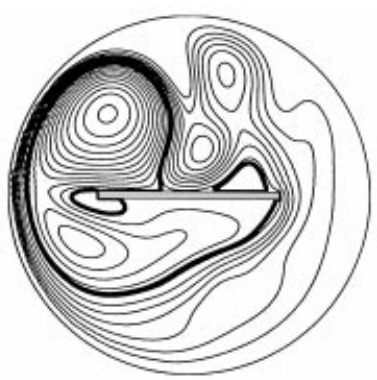

(d)

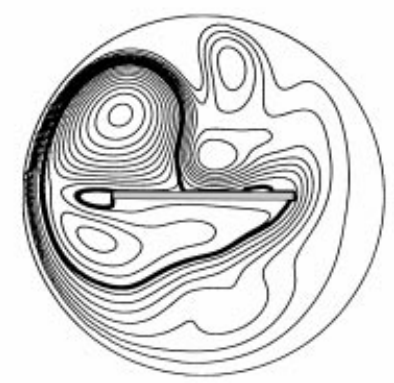

FIGURE 14. The streamfunction for $\delta_{I} / \delta_{M}=2.56$ at four times: (a) $t=4.88,(b) 5.26,(c) 5.64$ and (d) 6.01 . North is at the top of each panel.

\section{Stagnation-point locations}

A summary of the mean southern stagnation point locations is plotted in Figure 8 (circles) along with the experimental results (squares). Again, once unsteadiness emerges a stagnation point is not well defined. For these cases the symbols give the mean position on the boundary where the instantaneous streamfunction with value equal to $\Psi_{I}$ intersects the island boundary. Since the flow on the southern side of the island is relatively steady and the transport fluctuations are small the point indicates the mean location of flow separation from the island. With this interpretation, the agreement between the experiments and numerical model results in figure 8 is quite good over the range of $\delta_{I} / \delta_{M}$ studied. For steady flows $\left(\delta_{I} / \delta_{M} \leqslant 1.63\right)$ the northern stagnation point resides at $\sim 0.62 \mathrm{~L}$ from the western tip of the island. This is in reasonable agreement with the experimental value of $\sim 0.58 \mathrm{~L}$. 



FIGURE 15. Transport around the island from the numerical model runs. (a) Transport $T$ normalized by $H \Psi_{I}$ from (9). (b) Transport in the western boundary layer, $T-\psi_{s}^{I}$, normalized by $H \Psi_{W}$ from (11). The circles are the mean transport from the numerical model; the range of variability indicated by the bars. The squares are the mean transports from the laboratory experiments shown in figure 9 .

\section{Transport}

The transport around the island, normalized by the Island Rule (9), is shown by the circles in figure 15. The bars show the magnitude of the variation from the mean transport (after the initial spin-up transients), which is quite small. In figure 15(a) the total transport is plotted and in figure $15(b)$ the transport in the western boundary layer is shown. The transport, relative to $\Psi_{I}$ or $\Psi_{W}$, decreases slightly as $\delta_{I} / \delta_{M}$ increases above one, but the effects of nonlinear processes are minimal. Again the linear theory for $\Psi_{I}$ is a robust predictor of the transport. The mean laboratory transports from figure 9 are plotted along with the numerical results in figure 15.

\section{Tilted island}

Lastly, the flow around a thin island which lies at an arbitrary angle $\theta$ from zonal orientation is considered. The first issue which arises is the applicability of the modified Island Rule for Munk dynamics, (9). A thin island tilted at a finite angle $\theta$ will now support a western boundary layer on the east side of the island. Following the scaling argument (2) the meridional extent of this boundary layer is $L \sin \theta$. The scale of the zonal boundary layer becomes $\delta_{Z}=\left(\delta_{M}^{3} L \cos \theta\right)^{1 / 4}$, where $L$ is again the total length of the island. The ratio of boundary dissipation influences is, following (2),

$$
R_{Z}=\frac{\left(\delta_{M}^{3} L \cos \theta\right)^{1 / 4}}{L \sin \theta}
$$

For $R_{Z} \ll 1$ the dissipation in the meridional boundary layer dominates. The transition, for small $\theta$, occurs at $\theta_{c} \sim\left(\delta_{M} / L\right)^{3 / 4}$. Using the value $\delta_{M} / L=0.032$ from the numerical calculations, $\theta_{c} \approx 0.08$. Very small rotations should switch the dominant dissipation mechanism to the western boundary layer on the eastern side of the island. The appropriate rule for the island streamfunction then reverts to the original formulation (7).

For the situation of a circular basin forced by a uniform wind stress curl and a centrally located island rotated about its midpoint, the streamfunction on the island can be found from (7) to be

$$
\Psi_{I}=\frac{W}{\beta L \sin \theta}\left[\frac{L}{2} \sin \theta\left(r_{0}^{2}-\left(\frac{L}{2} \sin \theta\right)^{2}\right)^{1 / 2}+r_{0}^{2} \sin ^{-1}\left(\frac{L}{2 r_{0}} \sin \theta\right)\right]
$$


(a)

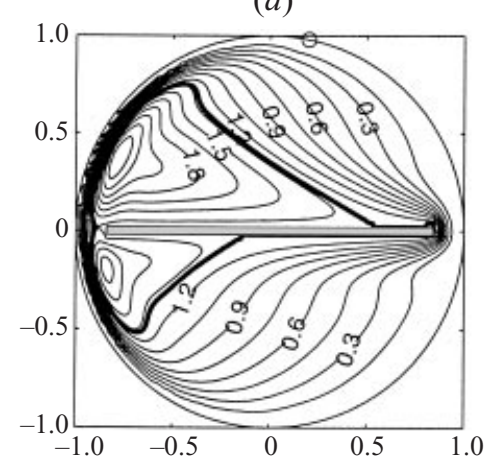

(b)

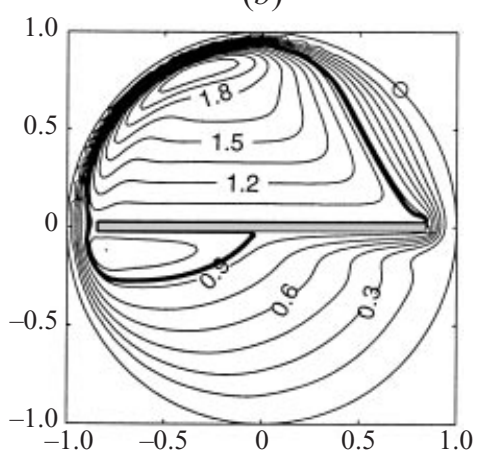

(c)

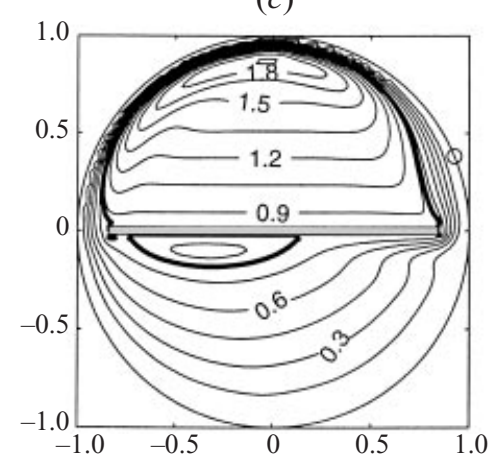

FIGURE 16. Streamfunction contours from the numerical model for rotated islands. $(a) \theta=0.2,(b)$ $\pi / 4$, (c) $3 \pi / 8$. In all the runs $\delta_{I} / \delta_{M}=0.1$. The circle on the basin boundary indicates the effective northward direction. The streamfunction is normalized by the Sverdrup streamfunction in the centre of the basin in the absence of the island and plotted in intervals of 0.15 . The thick contour has a value equal to the streamfunction on the island.

In deriving (21) the Sverdrup streamfunction for uniform wind stress curl in a circular basin,

$$
\psi_{s}=\frac{W}{\beta}\left(x-\left(r_{0}^{2}-y^{2}\right)^{1 / 2}\right)
$$

was used.

To test the scaling arguments and investigate the structural changes in the flow, several numerical model runs were conducted for nearly linear forcing $\delta_{I} / \delta_{M}=0.1$. The other parameters were the same as above except for the island orientation and length $L$, which was increased to $1.7 r_{0}$. The gaps are large enough to ignore frictional effects on the flow within them (PPSH). Also, the bottom friction coefficient $r$ was decreased by a factor of 2 so that the flow is more clearly in the Munk regime. Runs were conducted for island orientation from zonal, $\theta=0$, to meridional, $\theta=\pi / 2$. The island rotation was done by rotating $\nabla f$ so that the island boundaries remain aligned with the model grid.

Figure 16 shows the streamfunction fields for three runs $\theta=0.2, \pi / 4$ and $3 \pi / 8$. In these figures the circle on the basin boundary indicates the effective northward direction. The flow for $\theta=0$ (not shown) is similar to that shown in figure 3. For small rotations the flow is much like the purely zonal case but with a loss of meridional symmetry as shown in Figure $16(a)$ for $\theta=0.2$. The northern stagnation point has 


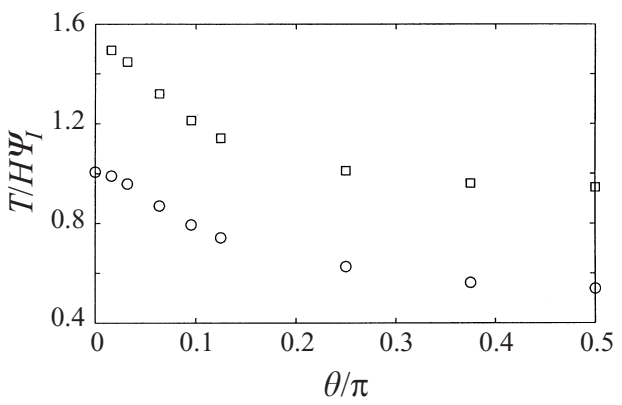

FIGURE 17. Transport, $T$ around the island versus rotation angle $\theta / \pi$. The circles show $T$ normalized by the rule for zonal islands (9) and the squares are transports non-dimensionalized by the rule for rotated islands (21).

moved towards the eastern end and the southern stagnation point has shifted to the west. Increasing $\theta$ results in movement of the northern stagnation point to the tip of the island (Figure 16b). The southern stagnation point remains near the centre of the island. The streamline emanating from this point defines a region of fluid which never touches the northern side of the island. Increasing $\theta$ further results in a recirculation cell on the eastern side of the island (Figure 16c). For $\theta=\pi / 2$ (not shown) the recirculation cell is symmetrically located on the eastern side of the island. This cell was discussed in detail in PPSH.

The transport around the island is shown in figure 17. In the figure the computed transports were normalized by the Island Rule for purely zonal islands (9) and plotted as circles, and by the Island Rule for tilted islands (21) and plotted as squares. The transport drops off rapidly as $\theta$ is increased, as expected from the purely zonal and meridional predictions. The figure shows that the zonal rule quickly breaks down for $\theta>0.05 \pi$. The transport is predicted well by the rule for rotated islands (21) for $\theta>0.2 \pi$. These results are in agreement with the scaling argument above.

\section{Discussion}

This study has extended the work in PPSH and the shadowed meridional island geometry in Helfrich et al. (1999) to consider flow around a zonally elongated island. The linear boundary layer theory developed in PPSH for a zonal island has been confirmed with laboratory experiments and numerical studies. In particular, it is found that for even moderately nonlinear flows, $\delta_{I} / \delta_{M} \leqslant 1.2$, the prominent features of the linear theory are maintained. These include stagnation points on either side of the island and an intense western boundary layer of vanishing meridional length on the eastern tip, though for moderate nonlinearity, the north-south symmetry of the linear solution is lost as the southern stagnation point moves to the east. The western boundary layer serves as a conduit for the eastward flowing fluid in the northern zonal boundary layer to round the tip of the island and enter the southern zonal boundary layer.

This unusual feature was found to persist even into the strongly nonlinear regime. However, in these cases the flow was unable to completely round the tip. The flow separated from the island. It is important to note that the flow did not separate by flowing east past the end of island. It turned south at the eastern tip, then separated to form a south-westward flowing jet. This separation was found to occur for $\delta_{I} / \delta_{M} \geqslant 1.63$ in both the laboratory and numerical results. The increased nonlinearity 
also resulted in the appearance of large eddies on the northern side of the island. For $\delta_{I} / \delta_{M}>2$ these eddies propagated to the eastern tip of the island, after which the eddy formation process repeated.

Both the experimental and the numerical results show the modified Island Rule for a thin barrier (9) to be a robust predictor of the mean transport around the island despite the significant structural changes and unsteadiness in the flow which occur with increasing nonlinearity. This is in agreement with the earlier results for single and multiple meridional barriers (PPSH; Helfrich et al. 1999).

While the modified Island Rule was a good predictor of the structure of the transport despite significant flow nonlinearity, the laboratory experimental and numerical transports were consistently smaller than the prediction. The differences may be due to several sources. First, the transports were scaled by the Munk version of the modified Island Rule (9) although the experiments were conducted for parameters where lateral and bottom friction were comparable. If instead the transports were scaled by the Stommel version of the Island Rule (10) the scaled transports in figures $9(a)$ and $15(a)$ should be increased by a factor of 1.11. This would bring the scaled numerical transports for $\delta_{I} / \delta_{M}<1.2$ to about 1.03. This slight overcorrection further indicates that the experiments are in a mixed boundary layer regime. Secondly, the theory ignores the viscous boundary layer on the eastern wall of the basin which if included would reduce the predicted transport. In Helfrich et al. (1999) the error was shown to be $O\left(\delta_{M} / \Delta_{e}\right)$, about $6 \%$ for the geometry studied here. This correction would pull the numerical results for $\delta_{I} / \delta_{M}<1$ into excellent agreement with the theory (see figure 15). The experiments show larger differences for $\delta_{I} / \delta_{M}<1$. This could be due to experimental error. For $\delta_{I} / \delta_{M}<1$ the flow is very slow $\left(U<0.01 \mathrm{~cm}^{-1} \mathrm{~s}^{-1}\right)$ and it takes over a minute for the dye swath to become large enough to permit accurate measurement. During this time the dye is subject to buoyancy effects which make the area swept by the dye difficult to measure accurately.

The minor reduction of mean transport compared to the theory for $\delta_{I} / \delta_{M}$ increasing above 1 may be due to increased dissipation near the sharp corners of the island. The linear theory for $\Psi_{I}$ is found from a boundary layer analysis which avoids the island corners. These $90^{\circ}$ corners, coupled with increased velocities, induce significant dissipation that is unaccounted for in the boundary layer theory but present in the numerical model and laboratory experiments (PPSH).

The numerical model was used to show that the streamfunction on islands rotated from the zonal direction quickly reverts to the original Island Rule for meridionally oriented islands. Rotations of order $20^{\circ}$ are all that are necessary because of the weakness of the zonal boundary layer dissipation in comparison to western boundary layers. Despite this restriction there are numerous topographic features in both the upper wind-driven and deeper abyssal flow that fall into the zonal category. Recall that the orientation is with respect to the ambient potential vorticity isolines which, particularly in the deep flow, may depart substantially from latitude circles (O'Dwyer \& Williams 1997). More difficult to estimate is the nonlinearity of the flow measured by $\delta_{I} / \delta_{M}$. This is due to a lack of data on the abyssal flow forcing (i.e. upwelling, or turbulent mixing, distribution) that sets the Sverdrup scale $U$, and very little understanding of the crucial dissipation mechanisms that set $\delta_{M}$. Despite these difficulties the results provide at least qualitative guidance for the interpretation of oceanographic observations and serve to highlight the important differences between zonal and western boundary effects in flow around planetaryscale islands. 
The authors thank John Salzig for the construction of the experimental apparatus and help in the laboratory. This work was supported by the National Science Foundation (Grant Number OCE96-16949). This is Woods Hole Oceanographic Institution Contribution Number 10112.

\section{REFERENCES}

BEARDSLey, R. C. 1969. A laboratory model of the wind-driven ocean circulation. J. Fluid Mech. 26, $255-271$.

Bleck, R., Rooth, C., Hu, D. \& Smith, L. T. 1992. Salinity-driven thermocline transients in a wind- and thermohaline-forced isopycnal coordinate model of the North Atlantic. J. Phys. Oceanogr. 22, 1486-1505.

GoDFREY, J. S. 1989. A Sverdrup model of the depth-integrated flow from the world ocean allowing for island circulations. Geophys. Astrophys. Fluid Dyn. 45, 89-112.

Griffiths, R. W. \& Kiss, A. E. 1999. Flow regimes in a wide 'sliced-cylinder' model of homogeneous beta-plane circulation. J. Fluid Mech. 399, 205-236.

HarT, J. E. 1995. Nonlinear Ekman suction and ageostrophic effects in rapidly rotating flows. Geophys. Astrophys. Fluid Dyn. 79, 201-222.

Helfrich, K. R., Pedlosky, J. \& Carter, E. 1999. The shadowed island. J. Phys. Oceanogr. 29, 2559-2577.

O'Dwyer, J. \& Williams, R. G. 1997. The climatalogical distribution of potential vorticity over the abyssal ocean. J. Phys. Oceanogr. 27, 2488-2506.

Pedlosky, J. 1987. Geophysical Fluid Dynamics. Springer-Verlag, 710pp.

Pedlosky, J. 1996. Ocean circulation theory. Springer-Verlag, 450pp.

Pedlosky, J. \& Greenspan, H. P. 1967. A simple laboratory model for the oceanic circulation. $J$. Fluid Mech. 27, 291-304.

Pedlosky, J., Pratt, L. J., Spall, M. A. \& Helfrich, K. R. 1997. Circulation around islands and ridges. J. Mar. Res. 55, 1199-1251 (referred to herein as PPSH).

Pratt, L. J. \& Pedlosky, J. 1998. Barotropic circulation around islands with friction. J. Phys. Oceanogr. 28, 2148-2162.

Wajsowicz, R. C. 1993. The circulation of the depth-integrated flow around an island with application to the Indonesian throughflow. J. Phys. Oceanogr. 23, 1470-1484. 